\title{
Aislamiento acústico entre recintos superpuestos de gran volumen con forjados autárquicos
}

\author{
Sound insulation between overlapping large volume rooms with thin single direction slabs.
}

\author{
David Caballol (Autor Principal y Autor Oficial de Contacto) \\ david.caballol@upm.es \\ UPM, ETSEM, Departamento de Construcciones Arquitectónicas \\ y su Control, España \\ Fono +34 913367596 Fax +34 913367634 \\ Avda. Juan de Herrera, 628040 Madrid
}

\author{
Cesar Díaz \\ UPM, España \\ cesar.diaz.sanchidrian@upm.es
}

\section{Resumen}

En este trabajo se exponen y se analizan los resultados de las mediciones acústicas del aislamiento a ruido aéreo y a ruido de impactos entre recintos superpuestos con forjados unidireccionales de pequeño espesor $(12-14 \mathrm{~cm})$ construidos hasta los primeros años de la década de 1960 en recintos de volumen superior a $250 \mathrm{~m}^{3}$. Se han comparado los valores experimentales obtenidos del índice ponderado de reducción acústica aparente $R^{\prime}{ }_{w}$ y nivel de presión acústica ponderado de impactos normalizado $L_{n, w}^{\prime}$ con los valores previstos en los modelos de cálculo de las normas UNE EN 12354 partes 1, 2:2000. Como conclusión, con los resultados experimentales se ha obtenido una relación de reciprocidad para la suma de los valores del índice de reducción acústica aparente y el nivel de presión sonora de impactos normalizado.

\author{
Antonio Rodríguez \\ UPM, España \\ antonio.rodriguezs@upm.es \\ Eduardo Medina \\ UPM, España \\ eduardo.medina@upm.es
}

Código: 0179
Fecha de Aceptación: 1 diciembre 2013

Abstract

This paper presents and analyses the results of acoustic measurements of the airborne sound insulation and impact noise between overlapping rooms with thin $(12-14 \mathrm{~cm})$, single direction overlaid slabs, built up to the early 1960s in rooms over $250 \mathrm{~m}^{3}$. The experimental values for the acoustic parameters are as follows: weighted apparent sound reduction index $R^{\prime} w$ and weighted normalized impact sound pressure level $L^{\prime}{ }_{n, w}$ have been compared with the one predicted by the estimations in the calculation models in the standards UNE EN 12354 parts 1, 2:2000. As a conclusion, an experimental reciprocity relation was obtained for the addition of the values of the apparent sound reduction index and the normalized impact sound pressure level.

\section{Introducción y descripción del problema}

Tras la guerra civil española (1936-1939), la situación constructiva del país hubo de adaptarse al escenario de la escasez y el aislamiento internacional. Fueron habituales las dificultades en el suministro de todo tipo de materiales, con una industria interior muy deteriorada por los efectos de la guerra y escasas posibilidades de obtener materiales importados. Para tratar de paliar el problema de la escasez de materiales de construcción y sobre todo de acero, en 1943 se dictó la Orden "sobre preferencia de suministro de cemento a construcciones con ahorro de hierro" (Ministerio de la Presidencia, 1943) en la que se daba preferencia a "edificios de cualquier orden, cuya construcción se efectúe con arreglo a sistemas por cuya aplicación se reduzca el consumo de elementos metálicos".

El sector del cemento estuvo oficialmente intervenido, a través de la "Delegación del Gobierno en la Industria del Cemento" (DGIC) que desde 1941 y hasta 1961 se encargó de fijar los precios, los cupos de entrega obligatorios por parte de las fábricas, y los cupos de adjudicación del cemento. En varios forjados de los edificios que se reconstruyeron y se iniciaron en los años 40 y 50 del pasado siglo en la Ciudad Universitaria de Madrid, se mantuvieron y repararon las losas macizas de hormigón armado con espesores reducidos $(12-14 \mathrm{~cm})$ (Ramírez, Bárcena y Landa, 1990), pero la escasez de acero hizo que la construcción pronto evolucionara hacia soluciones de forjados de pequeño espesor (Paricio. 1996), habitualmente conocidos a causa de las condiciones económicas de su época como forjados autárquicos.

El arquitecto y catedrático D. Ignacio Paricio Ansuategui al respecto describió: "Las necesarias limitaciones en el uso de elementos metálicos durante la posguerra llevan el forjado a su momento más bajo de calidad. La cantidad de acero utilizado se convierte en una obsesión y agudiza el ingenio buscando soluciones cada vez más apuradas para arañar algunos gramos de material en cada metro cuadrado. En ese momento se difunden los forjados cerámicos que optimizan el uso del acero utilizado exclusivamente allí donde es insustituible: para soportar las tracciones... Los años cincuenta nos muestran el límite de este angustioso proceso de reduccionismo: el forjado de vigueta pretensada. El techo, reducido a su más elemental función: transmitir hasta los pilares las cargas verticales merced a su capacidad de soportar flexiones, se convierte en una sucesión de viguetas de apuradísimo cálculo, entre las que solo cabe un entrevigado de bovedilla cerámica que apenas deja huecos para un escueto vertido de hormigón. Sin capa de compresión, sin zuncho, sin peso, con unas viguetas producidas por unos fabricantes sobre cuya picaresca de pretensar se ha dicho todo, el forjado ha tocado fondo en su evolución".

El proceso de ahorro dirigido y de intervención estatal se truncó en 1959 con la llegada del plan de estabilización. A partir de entonces y según fue progresando el ciclo de crecimiento económico en la década de los años 60, los sistemas constructivos fueron relajando los requisitos de ahorro material 
e incrementando sus controles y requisitos de calidad. Se han realizado mediciones acústicas en siete recintos superpuestos con forjados autárquicos como elemento separador, mediante aplicación de las normas UNE EN ISO 140 -Partes 4, 7 y 14 y UNE EN ISO 717- Partes 1 y 2.

Como magnitud relevante para expresar el aislamiento a ruido aéreo entre recintos se ha empleado el índice de reducción acústica aparente $R^{\prime}$, calculado de las mediciones acústicas realizadas in situ de acuerdo con la fórmula expresada en la norma anteriormente citada. Donde $L_{1}$ y $L_{2}$ son los niveles de presión sonora promedios medidos en los recintos emisor y receptor respectivamente, en $\mathrm{dB}, \mathrm{S}$ es el área del elemento separador entre recintos, en $\mathrm{m}^{2}$ y $\mathrm{A}$ es el área de absorción acústica equivalente en el recinto receptor, en $\mathrm{m}^{2}$.

En la evaluación del nivel de ruido de impactos entre los recintos se ha utilizado el nivel de presión sonora de impactos normalizado L'n, obtenido de la expresión definida en la norma UNE EN ISO 140 -Parte 7. Donde $L_{i}$ es el nivel de presión sonora de impactos en bandas de frecuencia, en tercios de octava, medido en el recinto receptor, en $\mathrm{dB}$, cuando el suelo objeto de estudio está excitado por una máquina de impactos normalizada. A es el área de absorción acústica equivalente en el recinto receptor, en $\mathrm{m}^{2}$.

\section{Estado del arte: Los forjados en los recintos objeto de estudio}

El proyecto del edificio original de la Escuela Universitaria de Arquitectura Técnica de la UPM es obra del Arquitecto D. Pascual Bravo Sanfeliú y su construcción fue iniciada en el año 1959 y finalizada en 1962. Sus cerramientos exteriores están compuestos por dos hojas de fábrica. La hoja exterior, de un asta de espesor está realizada mediante fábrica de ladrillo hueco doble chapada con piedra arenisca Almorquí, y la cámara de aire se cierra con ladrillo hueco sencillo revestido interiormente con guarnecido y enlucido de yeso. La estructura interior del edificio es de hormigón armado con vigas $y$ soportes que se repiten sobre una trama regular con vigas de cuelgue de $30 \mathrm{~cm}$ de ancho y $80 \mathrm{~cm}$ de canto y una distancia al eje del entrevigado de $320 \mathrm{~cm}$. Sobre el forjado se encuentra un pavimento adherido de terrazo in situ, con tan solo $3 \mathrm{~cm}$ de espesor y la parte inferior se encuentra guarnecida y enlucida con $1,5 \mathrm{~cm}$ de yeso. Los forjados objeto de estudio cumplen con todas las características de los forjados autárquicos descritos anteriormente y se encuentran en el edificio original de la Escuela Universitaria de Arquitectura Técnica de la UPM en la Ciudad Universitaria de Madrid, España. Se verificó su tipología mediante la realización de catas, una de las cuales, una vez realizada y documentada, se destacó para emplearla con fines docentes y actualmente puede ser observada (Imagen 1) dentro del Aula Museo de la misma Escuela.
Figura 1. Cata inferior realizada en el forjado. Elaboración propia, 2013.

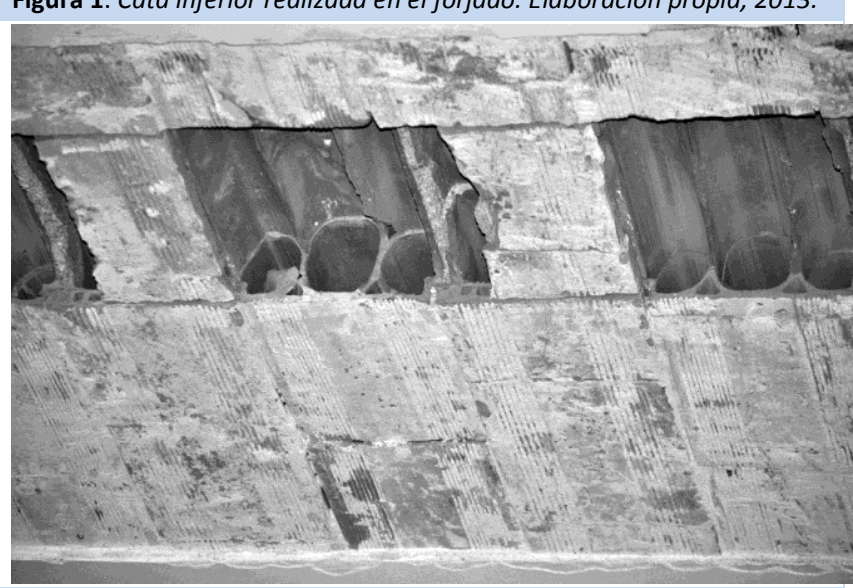

Los forjados son unidireccionales con un espesor de $12 \mathrm{~cm}$ y fueron realizados mediante la disposición de viguetas cerámicas elaboradas in situ con un entrevigado de bovedillas cerámicas. Su masa superficial es tan solo de $110 \mathrm{~kg} / \mathrm{m}^{2}$ (Ministerio de la Gobernación, 1940). La densidad del hormigón armado empleado es de $2.400-2.500 \mathrm{Kg} / \mathrm{m}^{3}$ y el acero empleado, liso, sin corrugas. La masa promedio por unidad de superficie del forjado con las vigas de cuelgue es de aproximadamente $350 \mathrm{~kg} /{ }^{\mathrm{m} 2}$. Todos los forjados estudiados se encuentran dispuestos entre vigas de cuelgue de hormigón armado y debido a su pequeño espesor, son adecuados para salvar luces limitadas. No disponen de capa de compresión y sobre los mismos se dispone el pavimento de terrazo in situ de tan solo $3 \mathrm{~cm}$ de espesor mencionado anteriormente. En la figura 2 se muestra un detalle constructivo del forjado objeto de estudio.

Figura 2. Detalle constructivo del forjado objeto de estudio. Elaboración propia, 2013.

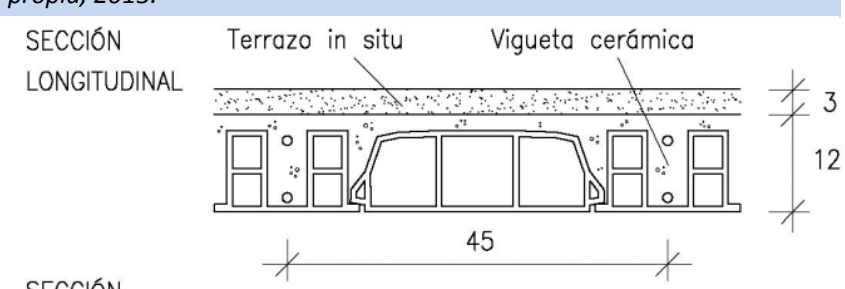

SECCIÓN

TRANSVERSAL Forjado

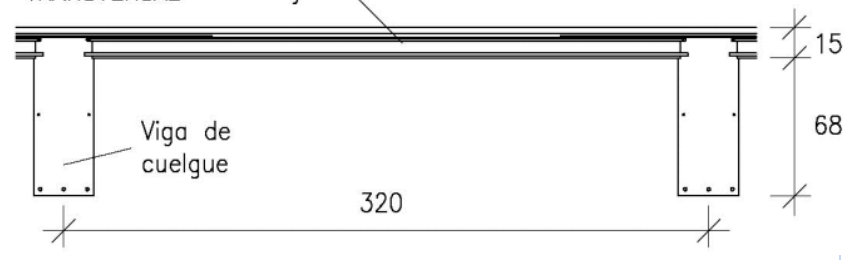

Los recintos estudiados mantienen sin apenas modificaciones la volumetría original con la que fueron diseñados. Las modificaciones realizadas en su interior han sido tan solo de mobiliario, continúan con el pavimento original y sin disponer de falso techo. En la Tabla 1 se muestran las dimensiones geométricas de los recintos superpuestos estudiados. $\mathrm{El}$ área común de separación horizontal entre recintos es en todos los casos superior a $100 \mathrm{~m}^{2}$ y el volumen de alrededor de $400 \mathrm{~m}^{3}$. 
Tabla 1. Datos geométricos de los recintos estudiados. Elaboración propia 2013.

Ensayo Recintos Largo $m$ Ancho m Alto m Volumen

$\mathrm{m}^{3}$ Área superficie común, $\mathrm{m}^{2}$

\begin{tabular}{lllllll}
\hline \multirow{2}{*}{ A1/I1 } & P0.A1 & 12,53 & 8,95 & 3,55 & 397,9 & \multirow{2}{*}{112,1} \\
& S1.A1 & 12,53 & 8,95 & 3,56 & 399,2 & \\
\hline \multirow{2}{*}{ A2/I2 } & P0.A2 & 12,53 & 8,95 & 3,55 & 397,9 & \multirow{2}{*}{112,1} \\
& S1.A2 & 12,53 & 8,95 & 3,56 & 399,2 & \\
\hline \multirow{2}{*}{ A3/I3 } & P0.A3 & 12,53 & 8,95 & 3,55 & 397,9 & \multirow{2}{*}{112,1} \\
& S1.A3 & 12,53 & 8,95 & 3,56 & 399,2 & \\
\hline \multirow{2}{*}{ A4/14 } & P1.A1 & 12,53 & 8,95 & 3,54 & 396,8 & \multirow{2}{*}{112,1} \\
& P0.A1 & 12,53 & 8,95 & 3,55 & 397,9 & \\
\multirow{2}{*}{ A5/15 } & P1.A2 & 12,53 & 8,95 & 3,54 & 396,8 & \multirow{2}{*}{112,1} \\
& P0.A2 & 12,53 & 8,95 & 3,55 & 397,9 & \\
\multirow{2}{*}{ A6/16 } & P2.A2 & 12,53 & 8,95 & 3,58 & 409,6 & \multirow{2}{*}{112,1} \\
& P1.A2 & 12,53 & 8,95 & 3,54 & 396,8 & \multirow{2}{*}{112,1} \\
\hline \multirow{2}{*}{ A7/17 } & P2.A3 & 12,53 & 8,95 & 3,58 & 409,6 & \\
& P1.A3 & 12,53 & 8,95 & 3,54 & 396,8 & \\
\hline
\end{tabular}

Metodología y resultados experimentales

Las dimensiones de los recintos ensayados en todos los casos son mayores que las de los recintos habituales en los edificios de uso residencial, por lo que siguiendo las indicaciones de las normas UNE EN ISO 140 - Partes 4, 7 y 14, con la intención de mejorar la reproducibilidad de las mediciones in situ, fue necesario utilizar en las mediciones acústicas del aislamiento a ruido aéreo, tres posiciones de fuente sonora, quince posiciones para las medidas de $L_{1}$ y $L_{2}$, y 24 posiciones para la medida del tiempo de reverberación T2. En el caso de la máquina de impactos se emplearon 8 posiciones. El tiempo de promediado de cada medida fue de 30 segundos. En las Figuras 3 y 4 se muestran los resultados experimentales de las mediciones del índice de reducción acústica aparente $R^{\prime}$ y del nivel de presión sonora de impactos normalizado L'n en el rango de frecuencias de un tercio de octava entre $100 \mathrm{~Hz}$ y $5000 \mathrm{~Hz}$.

\section{Análisis de los resultados obtenidos}

\section{Aislamiento a ruido aéreo}

Las mediciones acústicas del índice de reducción acústica aparente entre los siete recintos ensayados muestran que la frecuencia crítica de coincidencia está situada en la banda de tercio de octava de frecuencia central $100 \mathrm{~Hz}$ y que el valor medio del índice ponderado de reducción acústica aparente es $\mathrm{R}_{\mathrm{w}}{ }_{\mathrm{W}}(\mathrm{C} ; \mathrm{Ctr})=49(-1 ;-4) \mathrm{dB}$. En la tabla 2 se puede comprobar que la desviación típica de los valores de $R^{\prime}$ varía entre 0,46 y 1,81 $\mathrm{dB}$ y que el valor medio de la desviación típica de los valores de $R^{\prime}$ es $0,85 \mathrm{~dB}$. En la figura 3 se pueden observar las pequeñas diferencias entre los resultados obtenidos en cada una de las bandas de frecuencia en los ensayos realizados.

La norma UNE EN 12354-1 proporciona un método predictivo mediante la aplicación de una ley de masa teórica que nos permite calcular el índice ponderado de reducción acústica $R_{w}$. Esta ley de masa está basada en ensayos realizados durante varias décadas en distintos laboratorios europeos y calcula el índice ponderado de reducción acústica a partir de la masa por unidad de área $\mathrm{m}^{\prime}$ del conjunto constructivo solidario. Se considera que la masa superficial del forjado no es la única que se opone a la vibración mecánica forzada por la fuerza de excitación de las ondas acústicas, sino que es el conjunto de las masas unidas rígidamente (en este caso forjado autárquico + estructura horizontal + pavimento adherido + guarnecido y enlucido de yeso). El conjunto se comporta como una placa de espesor constante de aproximadamente $16 \mathrm{~cm}$, con una masa superficial media de $350 \mathrm{~kg} / \mathrm{m}^{2}$.

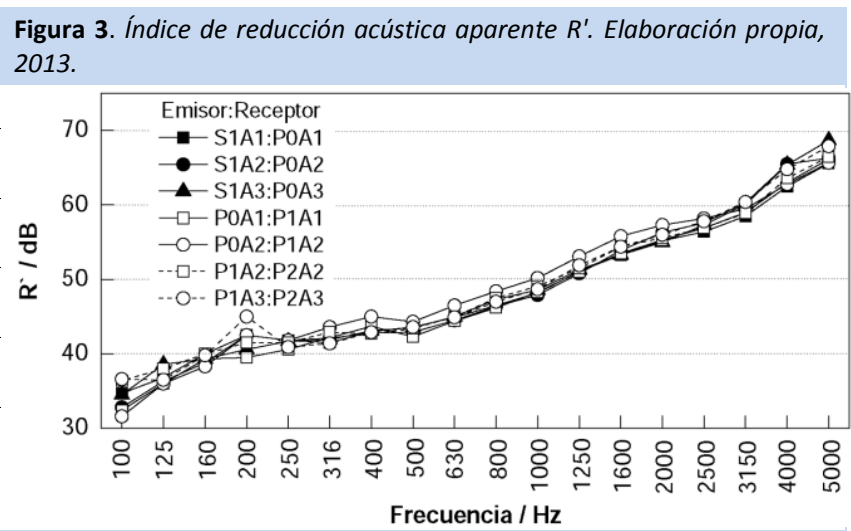

Figura 4. Nivel de presión sonora de impactos normalizado L'n. Elaboración propia, 2013.

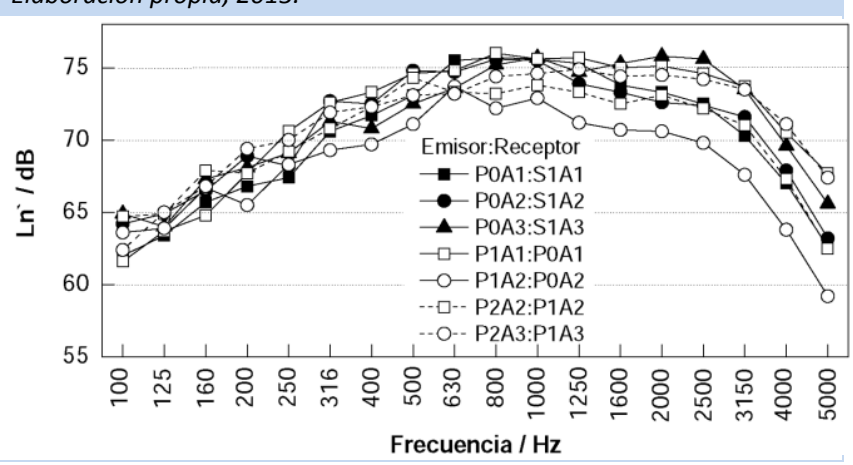

Al sustituir la masa superficial conjunta en la ecuación anterior se obtiene el valor de $R_{w}=53 \mathrm{~dB}$ para el sistema de forjado autárquico con vigas de cuelgue de hormigón armado y terrazo in situ. La diferencia entre el valor teórico en laboratorio y los valores experimentales in situ es de aproximadamente $4 \mathrm{~dB}, \mathrm{y}$ está producida por las transmisiones indirectas a través de las paredes, cuyo estudio más a fondo, necesitaría de un estudio experimental del índice de reducción de vibraciones, Kij. (Del Rey et al., 2012). De acuerdo con los resultados obtenidos por Díaz y Pedrero (2004) el índice ponderado de reducción acústica aparente $R^{\prime}{ }_{w}$ se obtiene mediante la ecuación [1]. Al sustituir el valor de la masa por unidad de superficie en la ecuación anterior, en el caso de los forjados autárquicos se obtiene $R^{\prime}{ }_{w}=49 \mathrm{~dB}$, valor que coincide con los resultados obtenidos experimentalmente.

Tabla 2. Promedios y desviaciones típicas de los distintos índices. Elaboración propia, 2013

\begin{tabular}{ccccccc}
\hline \multirow{2}{*}{$\begin{array}{c}\text { Frecuencia } \\
\text { Hz }\end{array}$} & \multicolumn{2}{c}{$\mathbf{R}^{\prime} \mathbf{d B}$} & \multicolumn{2}{c}{$\mathbf{L}_{n}{ }_{n}, \mathbf{d B}$} & \multicolumn{2}{c}{$\mathbf{R}^{\prime}+\mathbf{L}^{\prime}{ }_{n}, \mathbf{d B}$} \\
\cline { 2 - 7 } & Promedio & $\begin{array}{c}\text { Desviación } \\
\text { Típica }\end{array}$ & Promedio & $\begin{array}{c}\text { Desviación } \\
\text { Típica }\end{array}$ & Promedio & $\begin{array}{c}\text { Desviación } \\
\text { Típica }\end{array}$ \\
\hline 100 & 34,1 & 1,81 & 63,3 & 1,24 & 97,5 & 2,31 \\
125 & 36,9 & 0,94 & 64,3 & 0,61 & 101,1 & 1,19 \\
160 & 39,4 & 0,59 & 66,5 & 0,95 & 105,9 & 1,16 \\
\hline 200 & 42,0 & 1,61 & 67,7 & 1,20 & 109,7 & 2,25 \\
250 & 41,4 & 0,46 & 69,0 & 1,02 & 110,4 & 0,64 \\
315 & 42,2 & 0,72 & 71,3 & 1,12 & 113,5 & 0,91 \\
\hline 400 & 43,3 & 0,75 & 71,8 & 1,11 & 115,1 & 0,88 \\
500 & 43,4 & 0,58 & 73,4 & 1,22 & 116,7 & 0,78 \\
630 & 45,0 & 0,64 & 74,1 & 0,82 & 119,1 & 0,83 \\
\hline
\end{tabular}




\begin{tabular}{ccccccc}
\hline 800 & 47,0 & 0,71 & 74,6 & 1,32 & 121,7 & 0,71 \\
1000 & 48,7 & 0,70 & 74,8 & 1,02 & 123,5 & 0,46 \\
1250 & 51,5 & 0,72 & 74,1 & 1,42 & 125,7 & 0,97 \\
\hline 1600 & 54,1 & 0,82 & 73,6 & 1,48 & 127,7 & 0,87 \\
2000 & 55,8 & 0,74 & 73,6 & 1,61 & 129,4 & 1,07 \\
2500 & 57,3 & 0,57 & 73,0 & 1,78 & 130,3 & 1,68 \\
\hline 3150 & 59,5 & 0,70 & 71,6 & 2,06 & 131,1 & 2,39 \\
4000 & 63,9 & 1,18 & 68,2 & 2,31 & 132,1 & 3,05 \\
5000 & 66,7 & 1,08 & 64,0 & 2,82 & 130,7 & 3,56 \\
\hline
\end{tabular}

sonora de impactos normalizado depende únicamente de la frecuencia (Heckl y Rathe, 1963) (Cremer y Heckl, 1988). La relación de reciprocidad en bandas de frecuencia de octava sigue la siguiente expresión [3]. Siendo $f$ la frecuencia central de la banda de octava en hercios. Se puede deducir de la ecuación anterior [3], que la relación entre los valores globales ponderados es la mostrada en ecuación [4]. De acuerdo con los resultados obtenidos por Díaz y Pedrero (2004), la suma de los valores globales ponderados in situ viene dado por la ecuación [5]. Los valores experimentales obtenidos en este trabajo

$$
R_{w}^{\prime}=29+8 \log \left(\frac{m^{\prime}}{1 \mathrm{~kg} / \mathrm{m}^{2}}\right) d B
$$

$[1]$

$$
L_{n, w}^{\prime}=115-15 \log \left(\frac{m^{\prime}}{1 \mathrm{~kg} / \mathrm{m}^{2}}\right) d B
$$

\section{Nivel de ruido de impactos}

Las mediciones acústicas del nivel de presión acústica de impactos normalizado $L_{n, w}^{\prime}(\mathrm{Cl})$ varían entre $76(-9) \mathrm{dB}$ y $81(-11)$ $\mathrm{dB}$ y el valor medio del nivel de presión acústica ponderado de impactos normalizado es $L_{n, w}^{\prime}(C l)=79(-10) d B$. En la tabla 2 se puede comprobar que la desviación típica de los valores de $L^{\prime}{ }_{n}$ varía entre 0,61 y $2,82 \mathrm{~dB}$ y que el valor medio de la desviación típica de los valores de $L_{n}$ es $1,40 \mathrm{~dB}$. En la figura 4 se puede observar que las curvas que representan los valores experimentales de $L_{n}{ }_{n}$ son muy parecidas entre sí, presentando una mayor dispersión a medias y altas frecuencias. Como en el caso del aislamiento acústico a ruido de impactos, la norma UNE EN 12354-2 plantea una metodología predictiva simplificada mediante la aplicación de una ley de masa teórica que nos permite calcular el nivel normalizado de presión sonora de impactos en recintos superpuestos separados por un elemento constructivo horizontal considerado homogéneo, por lo que es posible su aplicación a nuestros recintos. La fórmula expresada en la UNE EN12354-2 utiliza el término $\mathrm{K}$ como corrección por la transmisión de ruidos de impacto sobre las construcciones de flancos homogéneas en decibelios. En los siete casos de recintos ensayados la corrección es $\mathrm{K}=1 \mathrm{~dB}$.

Al sustituir en la ecuación anterior se calcula un nivel normalizado de presión sonora de impactos, su valor es $L_{n, w}^{\prime}=$ $74 \mathrm{~dB}$. La diferencia entre los valores promedios medidos y los calculados es de $5 \mathrm{~dB}$. De acuerdo con los resultados obtenidos por Gerretsen (1985) el nivel normalizado de presión sonora de impactos $L_{n, w}^{\prime}$ se obtiene mediante la ecuación [2]. Al sustituir el valor de la masa por unidad de superficie en la ecuación anterior, en el caso de los forjados autárquicos se obtiene $L_{n, w}^{\prime}$ $=77 \mathrm{~dB}$, valor que difiere en $2 \mathrm{~dB}$ con los resultados obtenidos experimentalmente.

\section{Relación entre índices}

En laboratorio, para el caso de sistemas constructivos homogéneos y sin tener en cuenta las transmisiones forzadas, los aislamientos a ruido aéreo y a ruido de impactos están relacionados mediante una ley de reciprocidad, de modo que la suma del índice de reducción acústica y el nivel de presión cumplen con lo previsto en la ecuación [5].

Con objeto de obtener una relación de reciprocidad en bandas de octava para la tipología de forjado forjados autárquicos descrita con anterioridad, se realiza en primer lugar una regresión múltiple en la cual se analizan como variable dependiente los valores suma de los índices de reducción acústica aparente y niveles de presión sonora de impacto normalizado $\left(L_{n}+R^{\prime}\right)$ y como variables independientes, el logaritmo decimal de la frecuencia y un código marcador de cada una de las parejas de recintos ensayados. En la regresión efectuada se pone de manifiesto que la variable código no tiene repercusión alguna ( $p$-valor $=0,72$ ) lo que demuestra la homogeneidad global de los datos y la coherencia de su estudio conjunto como regresión simple con dos variables $\left(L^{\prime}{ }_{n}+R^{\prime}\right)$ y el logaritmo decimal de la frecuencia.

$$
L_{n}+R=43+30 \log \left(\frac{f}{1 H z}\right) d B
$$

[3]

$$
R_{w}+L_{n, w}=126 \pm 2 d B
$$

[4]

$$
L_{n, w}^{\prime}=115-15 \log \left(\frac{m}{1 \mathrm{~kg} / \mathrm{m}^{2}}\right) d B
$$

[5]

Una vez realizada la regresión simple, basándose en los valores experimentales medidos in situ en bandas de octava de frecuencias centrales entre $125 \mathrm{~Hz}$ y $2000 \mathrm{~Hz}$, de la suma de sus índices de reducción acústica y niveles de presión sonora de impacto normalizado se puede obtener la siguiente ecuación experimental [6] para la tipología de forjados y vigas construidos mediante forjados unidireccionales de pequeño espesor mediante viguetas cerámicas in situ, bovedillas cerámicas y vigas de cuelgue.

$$
L_{n}^{\prime}+R^{\prime}=64+20 \log \left(\frac{f}{1 H z}\right) d B
$$

La suma de $R^{\prime}$ y $L^{\prime}{ }_{n}$ menos veinte veces el logaritmo decimal de la frecuencia en bandas de octava es una constante, llamada constante de reciprocidad. Para esta tipología de forjados obtenemos 64 como constante de reciprocidad. Si se comparan 
las variables de la ecuación obtenida con las de otras tipologías conocidas, el valor de la constante de reciprocidad es semejante pero ligeramente menor. Para el caso de forjados unidireccionales con vigas de hormigón y bovedillas cerámicas, con pavimento de terrazo o parquet en viviendas de uso residencial se obtiene un valor de la constante de reciprocidad de 66 (Díaz y Pedrero, 2004) y en los forjados de placa de hormigón macizo de $12 \mathrm{~cm}$ de espesor y vigas de cuelgue con pavimento de terrazo in situ, el valor de la constante de reciprocidad es 68 (Díaz et al., 2011). Sin embargo, la pendiente en todas las ecuaciones es exactamente la misma con independencia de su tipología.

En la figura 5 se comparan en bandas de octava, los valores de la suma de los índices de reducción acústica aparente y los niveles de presión sonora de impacto normalizado y los valores predichos mediante la ley de reciprocidad anteriormente expresada [6]. Se puede observar que el ajuste entre los valores ensayados y predichos es excelente.

Figura 5. Suma $L^{\prime} n+R^{\prime}$ ensayada y su ajuste a la ley de reciprocidad. Elaboración propia, 2013.

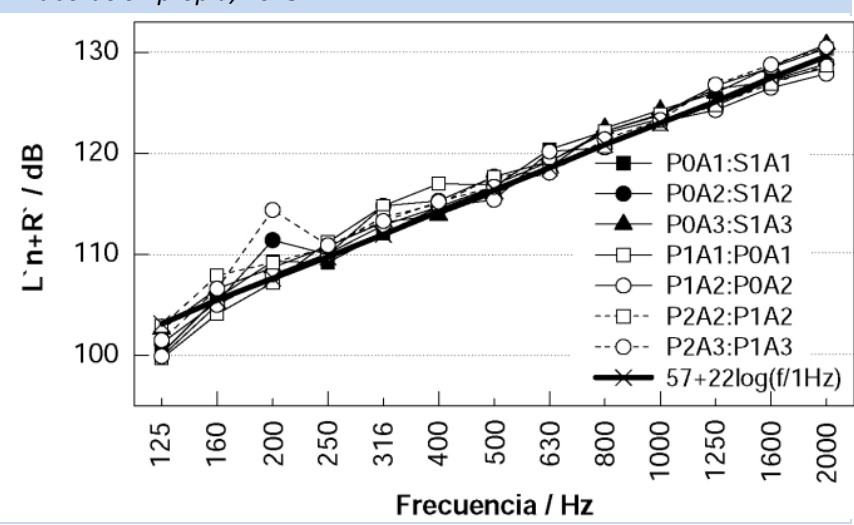

\section{Conclusiones}

Se muestran en este apartado algunas conclusiones sobre el comportamiento acústico de los forjados cerámicos aligerados de pequeño espesor fabricado in situ (forjados autárquicos), que se realizaron en España en la segunda mitad de los años 1940 y hasta la década de 1960. En estas fechas, como única normativa de referencia tan solo existía una relación de tipos de forjados aprobados y revisados por la Sección de Investigación y Normas, dependiente de la Dirección General de Arquitectura que regulaba la construcción de los mismos. Las conclusiones obtenidas en este estudio sobre el comportamiento acústico de este tipo de forjados son las siguientes:

Aunque el sistema de forjados cerámicos aligerados de pequeño espesor fabricado in situ con vigas de cuelgue es ortotropo, su comportamiento acústico a ruido aéreo y ruido de impactos se puede explicar de forma aproximada como el de un forjado homogéneo equivalente, empleando en los cálculos la masa superficial equivalente media. En los resultados de las mediciones del índice de reducción acústica aparente en función de la frecuencia se observa claramente el efecto de coincidencia en la banda de un tercio de octava de frecuencia central $100 \mathrm{~Hz}$, para frecuencias mayores el índice de reducción acústica aparente incrementa su valor en más de $6 \mathrm{~dB}$ por cada banda de octava. El valor en laboratorio del índice ponderado de reducción acústica $R_{w}$ previsto por la norma UNE EN 12354-1 difiere de los valores experimentales in situ del índice ponderado de reducción acústica aparente en aproximadamente $4 \mathrm{~dB}$. Esta diferencia está producida por las transmisiones indirectas a través de las paredes. En el caso del nivel de presión acústica ponderado de impactos normalizado $L_{n, w}^{\prime}$, la diferencia entre los valores medidos y los obtenidos según el modelo simplificado de la norma UNE EN 12354-2 es de $5 \mathrm{~dB}$.

Se ha obtenido una relación experimental de reciprocidad para la suma de los valores del índice de reducción acústica aparente y el nivel de presión sonora de impactos normalizado. Estos valores son semejantes a los obtenidos en el caso de recintos en viviendas de uso residencial con forjados de vigas de hormigón y bovedillas cerámicas con acabado de terrazo o parquet encolado, o en forjados de hormigón armado macizo fabricado in situ. Los valores obtenidos en los ensayos realizados de las magnitudes $R^{\prime}{ }_{w}, L_{n, w}^{\prime}, R^{\prime}{ }_{w}+L^{\prime}{ }_{n, w}$ así como la ley de reciprocidad, están dentro de los que se obtendrían por aplicación de las fórmulas propuestas para forjados con vigas de hormigón y bovedillas cerámicas con acabado de terrazo o parquet encolado. 


\section{Referencias}

Cremer, L. \& Heckl, M (1988). Structure Borne Sound. Berlin: Springer Verlag.

Del Rey, R, Alba, J, Ramis, J, y Bertó, L. (2012, Abr). Detección de puntos débiles de aislamiento acústico en edificación ya construida. Revista de la Construcción, 11(1), 75-86.

Díaz, C; Caballol, D y Díaz, A. (2011, Dic) La protección frente al ruido de los forjados proyectados por Eduardo Torroja en la E.T.S. de Arquitectura de la Ciudad Universitaria de Madrid. Informes de la Construcción, 524(63), 59-64.

Díaz, C.; Pedrero, A. (2004, Dec) Field measurements of Airborne and Impact Sound Insulation Between Rooms, One on Top of the Other, with Beam and Pot Floor Structures. Acta Acústica United with Acústica, 90(5), 982-986.

Gerretsen E. (1986, Jan) Calculation of airborne and sound insulation between dwellings. Applied Acoustics, 19(4), 245-264.

Heckl, M. \& Rathe, E. J (1963, Jan). Relationship between the transmission loss and the impact noise insulation of floor structures. JASA, 35(11), 1825-1830.

Ministerio de la Gobernación. Dirección General de Arquitectura. (1940). Sistemas especiales de forjados para la edificación, tipos aprobados y revisados por la Sección de Investigación y Normas. Madrid. (Autor anónimo)

BOE 336. 11569 (1943). Boletín Oficial del Estado. Orden del Ministerio de la Presidencia de 30 de noviembre de 1943. Madrid, España.

Paricio I. (1996) La Construcción de la Arquitectura 2. Los elementos. Barcelona, España: ITEC.

Ramírez, J.L; Bárcena J.M. y Landa P.J. (1990, Dic) Refuerzos de losas macizas de edificación en hormigón armado. Informes de la construcción, 410 (52), 65-74. 